Canadian

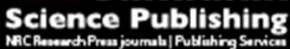

Applied Physiology, Nutrition, and Metabolism Physiologie appliquée, nutrition et métabolisme

\title{
Expectancy of ergogenicity from sodium bicarbonate ingestion increases high-intensity cycling capacity.
}

\begin{tabular}{|r|l|}
\hline Journal: & Applied Physiology, Nutrition, and Metabolism \\
\hline Manuscript ID & apnm-2015-0523.R1 \\
\hline Manuscript Type: & Article \\
\hline Date Submitted by the Author: & 05-Nov-2015 \\
\hline Complete List of Authors: & $\begin{array}{l}\text { Higgins, Matthew; Derby University, Sport, Outdoor and Exercise Science } \\
\text { Shabir, Akbar; Derby University, Sport, Outdoor and Exercise Science }\end{array}$ \\
\hline Keyword: & Psychobiology, Endurance, fatigue < exercise, RPE, Perceived Exertion \\
\hline & \multicolumn{2}{|l}{} \\
\hline
\end{tabular}


Title: Expectancy of ergogenicity from sodium bicarbonate ingestion increases high-intensity cycling capacity.

Running Head: Expectancy and sodium bicarbonate

Authors: Matthew F. Higgins and Akbar Shabir.

Author affiliations: Both authors are affiliated to Sport, Outdoor and Exercise Science, Department of Life Sciences, Derby University, Kedleston Road, Derby, DE22 1GB.

Corresponding Author:

Dr. Matthew F. Higgins: Department of Sport, Outdoor and Exercise Science, Derby University, Kedleston Road, Derby, DE22 1GB. Tel: +44 (0) 1332 591743 Email: m.higgins@derby.ac.uk 
Abstract

This study examined whether expectancy of ergogenicity of a commonly used nutritional supplement (sodium bicarbonate; $\mathrm{NaHCO}_{3}$ ) influenced subsequent high-intensity cycling capacity. Eight recreationally active males (age: $21 \pm 1$ years, body mass: $75 \pm 8 \mathrm{~kg}$, height: $178 \pm 4 \mathrm{~cm}, W_{\text {PEAK: }} 204 \pm 23 \mathrm{~W}$ ) performed a graded incremental test to assess peak power output ( $\mathrm{W}_{\text {PEAK }}$ ), one familiarisation trial and two experimental trials. Experimental trials consisted of cycling at 100\% $\mathrm{W}_{\text {PEAK }}$ to volitional exhaustion (TLIM) 60 min after

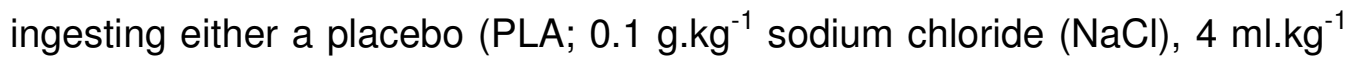
tap water and $1 \mathrm{ml}^{\mathrm{kg}}{ }^{-1}$ squash) or a sham placebo (SHAM; $0.1 \mathrm{~g} \cdot \mathrm{kg}^{-1} \mathrm{NaCl}$, $4 \mathrm{ml} . \mathrm{kg}^{-1}$ carbonated water and $1 \mathrm{ml}^{-\mathrm{kg}^{-1}}$ squash). SHAM aimed to replicate the previously reported symptoms of gut fullness (GF) and abdominal discomfort (AD) associated with $\mathrm{NaHCO}_{3}$ ingestion. Treatments were administered double blind and accompanied by written scripts designed to remain neutral (PLA) or induce expectancy of ergogenicity (SHAM). After

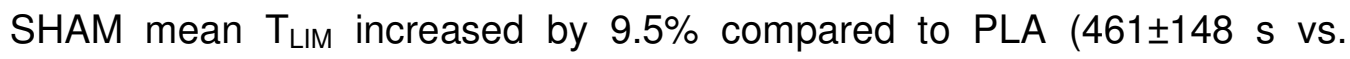
$421 \pm 150 \mathrm{~s} ; \mathrm{P}=0.048, \mathrm{~d}=0.3)$. Ratings of $\mathrm{GF}$ and $A D$ were mild but $\sim 1$ unit higher post ingestion for SHAM. After 3 min $T_{\text {LIM }}$ RPEO was $1.4 \pm 1.3$ units lower for SHAM compared to PLA $(P=0.020, d=0.6)$. There were no differences between treatments for blood lactate, blood glucose or heart rate. In summary, ergogenicity after $\mathrm{NaHCO}_{3}$ ingestion might be influenced by expectancy which mediates perception of effort during subsequent exercise. The observed ergogenicity with SHAM did not affect our measures of cardiorespiratory physiology or metabolic flux.

Keywords: Psychobiology, endurance, fatigue, RPE, perceived exertion 
Introduction

A significant amount of research has examined the efficacy of sodium bicarbonate $\left(\mathrm{NaHCO}_{3}\right)$ as an ergogenic aid (Cameron et al. 2010; Krustrup et al. 2015; Lavender and Bird 1989; Price and Simons 2010; Tan, et al. 2010). Cameron et al. (2010) reported that ingestion of $0.3 \mathrm{~g} \cdot \mathrm{kg}^{-1} \mathrm{NaHCO}_{3} 60$ min prior to exercise had no effect on multiple indices of repeated sprint performance in elite rugby players. Cameron et al. (2010) suggested the most likely reason for the lack of performance enhancement was due to the higher frequency and greater severity of gastrointestinal (Gl) symptoms after $\mathrm{NaHCO}_{3}$ ingestion. However, the lack of performance improvement might also have been influenced by the trained nature of participants with Peart et al. (2012) reporting that untrained individuals are significantly more likely to experience ergogenic benefit with $\mathrm{NaHCO}_{3}$. Indeed it is now well established that individuals who undertake high-intensity training have elevated levels of muscle carnosine compared with endurance trained and untrained individuals (Parkhouse and McKenzie 1984; Parkhouse et al. 1985). As carnosine is thought to play an important role in the homeostasis of muscle cells during high-intensity exercise (as an intracellular buffer) greater basal levels might 'offset' any potential ergogenic contribution from augmented extracellular $\mathrm{NaHCO}_{3}$ (Aschenbach et al. 2000; Derave et al. 2010).

In support of this, Higgins et al. (2013) reported that recreationally active healthy males who ingested $0.3 \mathrm{~g} \cdot \mathrm{kg}^{-1} \mathrm{NaHCO}_{3} 60$ min prior to cycling at $100 \% \mathrm{~W}_{\text {PEAK }}$ to volitional exhaustion $\left(\mathrm{T}_{\text {LIM }}\right)$ had on average $17 \%$ greater 
exercise capacity versus a taste matched placebo. Furthermore, the individual who had the highest increase in $\mathrm{T}_{\mathrm{LIM}}(+38 \%)$ also reported moderate-high Gl symptoms 30 min prior and immediately prior to exercise. The authors suggested that Gl symptoms might not necessarily negatively affect exercise performance, something that is supported by previous research (Price and Simons 2010).

According to Clark et al. (2000; pg 1642), the placebo effect is "...a favourable outcome arising purely from belief that one has received a beneficial treatment..." Expectancy is closely associated and in some instances assumed to have a direct relationship with the placebo effect (Kirsch 1985). It is suggested by enhancing the degree of expectancy, subsequently ones belief in the effectiveness of the placebo might increase (Evans 2003). The effects of expectancy on sport and exercise performance has been evaluated in a variety of different experimental models (Broatch et al. 2014; Maganaris et al. 2000; McClung and Collins 2007; Ross et al. 2015). For example, Maganaris et al., (2000) administered a saccharine placebo to eleven national level power lifters but provided false information to enhance expectancy of performance benefit. Participants were led to believe that they had consumed anabolic steroids which resulted in changes from baseline of $10 \pm 2 \mathrm{~kg}, 11 \pm 2 \mathrm{~kg}$ and $12 \pm 1 \mathrm{~kg}$, for maximal bench press, dead lift and squat performance, respectively. However, when approximately half of the group were told the true nature of the placebo these performance gains almost completely dissipated although remained for the group who believed they had consumed anabolic steroids (Maganaris, et al. 2000). 
To the best of our knowledge, only one study has previously evaluated the effects of expectancy on exercise performance with respect of $\mathrm{NaHCO}_{3}$ ingestion (McClung and Collins 2007). Sixteen trained endurance athletes ran $5 \times 1000 \mathrm{~m}$ time trials after ingesting either sodium bicarbonate $\left(\mathrm{NaHCO}_{3}\right)$ or a placebo in combination with being told correctly or deceived regarding what treatment had been administered (i.e. told $\mathrm{NaHCO}_{3} /$ placebo, given/not given $\mathrm{NaHCO}_{3} /$ placebo). The told $\mathrm{NaHCO}_{3} /$ given placebo treatment performed the time trial significantly faster $(-3.4 \mathrm{~s} /-1.8 \%)$ than the told placebo/given $\mathrm{NaHCO}_{3}$ treatment demonstrating the effects of expectancy on exercise performance were greater than the purely pharmacological effects of $\mathrm{NaHCO}_{3}$.

As there has been limited research on the potential psychobiological impact of $\mathrm{NaHCO}_{3}$ administration on exercise performance (McClung and Collins 2007) and based on previous data that suggests Gl distress does not necessarily negatively influence exercise performance (Higgins et al. 2013; Price and Simons 2010) we speculated that replicating side effects of $\mathrm{NaHCO}_{3}$ ingestion might initiate a degree of expectancy of ergogenicity which might influence exercise performance. Therefore, the aim of this study was to evaluate the effect of expectancy associated with $\mathrm{NaHCO}_{3}$ ingestion on high intensity cycling capacity. We hypothesised that by increasing the expectancy of ergogenicity a relatively inert compound $(\mathrm{NaCl})$ mimicked as closely as possible and described as $\mathrm{NaHCO}_{3}$ would induce a significant increase in cycling capacity. 
Materials and methods

Participants

Eight healthy and active male participants volunteered to take part in this study (age: $21 \pm 1$ years, body mass: $75 \pm 8 \mathrm{~kg}$, height: $178 \pm 4 \mathrm{~cm}$ ) which received university ethics committee approval. All participants were recreationally active although not specifically cycle trained ( $W_{\text {PEAK }}$ 204 \pm 23 W). In order to maximise the integrity of the treatment deception all participants were pre-screened to ensure they had not used alkalotic buffers for a minimum of 3 months. Furthermore, no participants had ever previously consumed $\mathrm{NaHCO}_{3}$ and only one had ever heard of its use with respect of sport and exercise performance.

Pre-experimental procedures

Participants were requested to avoid alcohol and strenuous exercise 12 and 24 hours prior to exercise, respectively. Participants were also requested to adopt the same balanced diet for 24 hours prior to each trial with adherence monitored via self-reported food diaries. Participants were specifically requested to avoid low carbohydrate intake which can induce mild metabolic acidosis and subsequently negatively affect exercise capacity (Greenhaff et al. 1988). The aforementioned information was included in the participant information sheet (PIS) and confirmed verbally before written consent was provided. Each participant completed physical activity readiness 
(PAR-Q) and blood screening questionnaires prior to the commencement of the study. Specifically, the blood screening questionnaire asked participants to report any serious infections, jaundice, haemophilia or anything that might be hazardous to the health of the participant or researcher. Individuals were only permitted to participate if they were deemed healthy and able to do so. Participants attended the laboratory at the same time of the day for each visit to minimise the influence of circadian changes on exercise performance (Fernandes et al. 2014). Trials were carried out 3 to 7 days apart.

Protocol

On the first visit to the laboratory, participants performed a graded incremental test to assess peak power output ( $\mathrm{W}_{\text {PEAK }}$ ) on a cycle ergometer (Ergomedic 884E 1 - Sprint Cycle, Monark, Sweden). Participants selected the seat and pedal strap positions that felt most comfortable with these settings being adopted for all subsequent trials. After 5 min seated rest a finger prick capillary blood sample was taken and analysed for blood lactate concentration ([BLa]) (Biosen C_line, EKF Diagnostic, Magdeburg, Germany). All participants' blood samples were analysed within $~ 10$ minutes of the participant leaving the laboratory.

Cycling commenced on the unloaded ergometer $(70 \mathrm{~W})$ at a cadence of 70 rev. $\mathrm{min}^{-1}$ with an increase of $35 \mathrm{~W}$ every 3 minutes until volitional exhaustion. Further blood samples were taken at the end of exercise and 5 min post-exercise. Exhaustion was assumed via voluntary stoppage or the 
cadence dropping below 70 rev. $\mathrm{min}^{-1}$ for longer than 5 seconds. Peak power output $\left(\mathrm{W}_{\mathrm{PEAK}}\right)$ was calculated as the mean power achieved during the final minute of the test (Lamberts et al. 2012). If exhaustion occurred less than one minute into a stage the appropriate duration undertaken at each power

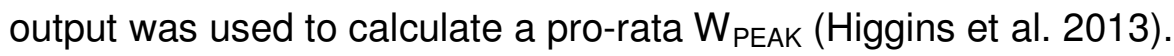

The second trial was a $\mathrm{T}_{\text {LIM }}$ familiarisation at $100 \% \mathrm{~W}_{\text {PEAK. After } 5 \mathrm{~min}}$ seated rest ratings of gut fullness (GF) and abdominal discomfort (AD) were recorded against an 11 point (0 to 10) scale with 0 representing 'empty' and 'completely comfortable', respectively and 10 representing 'bloated' and ‘unbearable pain', respectively (Higgins et al. 2013). A fingerpick capillary blood sample was then taken and analysed for [BLa] and blood glucose concentration [BG] (Biosen C_line, EKF Diagnostic, Magdeburg, Germany). Participants then ingested a control solution consisting of $1 \mathrm{ml} . \mathrm{kg}^{-1}$ double strength no added sugar orange squash with sweeteners (Cooperative Group, Manchester, UK) mixed with $4 \mathrm{ml}^{\mathrm{kg}}{ }^{-1}$ tap water in the first 5 min of a 60 min seated rest period with GF and AD recorded at the end of this 5 min period. Prior to commencing exercise (i.e. at end of 60 min rest), heart rate (HR; F1 Polar Heart Rate Monitor, Polar, Finland), GF and AD were recorded. Participants then warmed up for 3 minutes on the unloaded ergometer $(70 \mathrm{~W})$ at a cadence of $70 \mathrm{rev} \cdot \mathrm{min}^{-1}$. After a brief rest ( $\left.60 \mathrm{sec}\right)$ participants commenced exercise from a stationary start position and were counted down from 5 seconds (Wittekind et al. 2011). Exhaustion was assumed as previously described. Measures of AD and GF were recorded again at the end of exercise and 5 min post-exercise. Heart rate, ratings of 
perceived exertion specific to cardiovascular strain $\left(\mathrm{RPE}_{\mathrm{O}}\right)$ and specific to the leg musculature $\left(R P E_{L}\right)$ were recorded at 1 min intervals during exercise and at the end of exercise. The 15 point (6-20 category-ratio scale (CR-15) was used to assess RPEO which is deemed to have a linear relationship with work load, oxygen consumption and heart rate (Borg 1982). In contrast, the 10 point (1-10) category-ratio scale (CR-10) was used to assess $R_{P} E_{L}$. This approach has been shown to correlate better with sensations associated with lactate accumulation (McClung and Collins 2007). Further blood samples for $[B L a]$ and $[B G]$ were taken at the end of exercise and 5 min post-exercise. After final measurements had been collected and after completing a selfselected warm down participants left the laboratory.

On the third and fourth visits to the laboratory participants completed experimental trials which were identical to the aforementioned exercise protocol with the following amendments. Prior to treatment ingestion participants were provided with a written manuscript which detailed the proposed effects of the subsequent treatment. For PLA the manuscript suggested that $\mathrm{NaCl}$ was relatively inert and would have little impact on exercise performance, whereas for SHAM the 'proven' ergogenicity of $\mathrm{NaHCO}_{3}$ was presented and in line with the $\mathrm{AD}$ and GF scales adopted the potential for associated feelings of Gl distress such as build of gas and bloating were outlined. Participants then ingested either $0.1 \mathrm{~g}^{\mathrm{kg}}{ }^{-1}$ sodium chloride $(\mathrm{NaCl})$ mixed with orange squash as previously described and either $4 \mathrm{ml}^{\mathrm{kg}}{ }^{-1}$ tap water (placebo; PLA), or $4 \mathrm{ml}^{\mathrm{kg}}{ }^{-1}$ carbonated water (SHAM placebo; SHAM). Carbonated water was used for SHAM to differentiate from 
PLA and to attempt to induce mild Gl symptoms often associated with $\mathrm{NaHCO}_{3}$ ingestion such as gas/bloating. Control solutions demonstrated that the osmolality of PLA and SHAM were almost identical $\left(510 \pm 0 \mathrm{mOsmol} / \mathrm{kg}^{-1}\right.$ and $512 \pm 10 \mathrm{mOsmol} / \mathrm{kg}^{-1}$, respectively). Solutions were prepared by a technician not involved in data collection and both treatments and manuscripts were administered double blind. Trials were counterbalanced to minimise any potential order effects.

Statistical analysis

Statistical analysis was completed using SPSS (IBM v21, Chicago, USA). For all data normality (Shapiro-Wilk) and homogeneity of variance/sphericity (Mauchly) were checked prior to choosing the appropriate statistical tests. For 2-way repeated measures ANOVAs ([BLa], [BG]) Bonferroni corrections were applied. In some instances an ANOVA was chosen despite the majority of data not being normally distributed (i.e. AD and GF). This was decided so as to minimise the potential for type-l error due to multiple individual (non-parametric) comparisons and because of the general robustness of the univariate ANOVA test to violations of normality (Schmider et al. 2010). Additionally although $\mathrm{RPE}_{\mathrm{O}}, \mathrm{RPE}_{\mathrm{L}}$ and $\mathrm{HR}$ were collected during each minute of $\mathrm{T}_{\mathrm{LIM}}$, due to the variation in performance and small samples sizes at some time points analysis has only been completed between treatments for 1 to 7 minutes and at the end of exercise where at least $50 \%$ of data points were available for both treatments (minimum of $n=4$ comparison). With the exception of after 4 min $\mathrm{T}_{\text {LIM }}$, where a Mann-Whitney 
$\mathrm{U}$ test was used (for unequal sample size comparisons), $R P E_{O}, R P E_{L}$ and $H R$ were analysed by paired t-tests or Wilcoxon tests. Exercise capacity ( $\left.T_{\text {LIM }}\right)$ was analysed using a paired t-test.

Data were analysed and quantified using a mixture of effect sizes (ES), $P$ values (minimum $P \leq 0.05$ ) and, where appropriate, $95 \%$ confidence intervals (Watt et al. 2002). Unless otherwise stated data is presented as mean \pm standard deviation. For ANOVA main effects and interactions the ES is reported as the partial $\eta^{2}$ value. Otherwise, for normally distributed data the ES (d) was calculated using the difference in means divided by the pooled SD of the compared trials (Nakagawa and Cuthill 2007). 
Results

Exercise capacity

Mean TLIM increased by $9.5 \%(40 \pm 47 \mathrm{~s} ; 95 \% \mathrm{Cl}: 0.4$ to $80 \mathrm{~s})$ for SHAM vs. PLA (461 \pm 148 s vs. $421 \pm 150$ s; $P=0.048, d=0.3$; Figure 1$)$. No order effect was found between trials (448 \pm 130 s vs. $435 \pm 168 \mathrm{~s} ; P=0.574, d=0.1)$.

${ }^{\star * * *}$ Figure 1 near here ${ }^{* \star * *}$

Perceptual variables

There was no time and treatment interaction for $G F\left(P=0.276, \eta^{2}=0.2\right)$. Although GF increased by $~ 1$ unit at all time points post SHAM ingestion compared with PLA ingestion only a tendency towards a main effect for treatment was observed $\left(P=0.080 ; \eta^{2}=0.4\right)$. In contrast, there was a main effect for time $\left(P<0.001 ; \eta^{2}=0.7\right)$. More specifically, GF post-ingestion (1.7 \pm 1.1$)$ was greater than rest $(0.0 \pm 0.0 ; P=0.002 ; d=2.1)$, end of exercise (0.4 $\pm 1.1, \quad P=0.031 ; d=1.2)$ and $5 \mathrm{~min}$ post-exercise $(0.3 \pm 0.9, \quad P=0.012$; $\mathrm{d}=1.4)$. Pre-exercise, GF $(0.7 \pm 0.9)$ was also greater than 5 min post-exercise $(P=0.025 ; d=0.4 ;$ Figure 2). There was no time and treatment interaction for AD $\left(P=0.451, \eta^{2}=0.1\right)$. Similar to $G F$, although $A D$ increased by $\sim 1$ unit at all time points post SHAM ingestion compared with PLA ingestion, only a tendency towards a main effect for treatment was observed $(P=0.096$; 
$\left.\eta^{2}=0.3\right)$. Unlike GF there was no main effect for time for $A D(P=0.299$, $\left.\eta^{2}=0.2\right)$.

${ }^{* * * *}$ Figure 2 near here ${ }^{* * * *}$

After 3 min $T_{\text {LIM }}$ RPE $_{O}$ was $1.4 \pm 1.3$ units lower for SHAM (12.9 \pm 2.5$)$ units compared to PLA $(14.3 \pm 2.5 ; P=0.020, d=0.6)$. Although none of the other comparisons between treatments were significantly different, RPEO was also lower for SHAM vs. PLA after 2, 5, 6 and $7 \mathrm{~min}$ and at the end of exercise (Figure 3). There were no differences between treatments for $R P E_{L}$ although values were lower for SHAM vs. PLA after 3, 5, 6 and 7 min and at the end of exercise.

${ }^{\star \star \star \star}$ Figure 3 near here ${ }^{\star \star \star *}$

Blood and cardiorespiratory variables

There was no time and treatment interaction $\left(P=0.149, \eta^{2}=0.2\right)$ or main effect for treatment for $[B L a]\left(P=0.552, \eta^{2}=0.1\right)$. However, there was a main effect for time $\left(P<0.001, \eta^{2}=0.9\right)$. At the end of exercise $8.91 \pm 1.70$ $\left.\mathrm{mmol} . \mathrm{I}^{-1}\right)$ and $5 \mathrm{~min}$ post-exercise $\left(6.57 \pm 1.10 \mathrm{mmol}^{-\mathrm{I}^{-1}}\right)$ [BLa] was greater than at rest $\left(1.27 \pm 0.20 \mathrm{mmol}^{-1}{ }^{-1}\right.$; both $\mathrm{P}<0.001$ and $\left.\mathrm{d}>6\right)$. Additionally, [BLa] was lower 5 min post-exercise compared to the end of exercise $(P=0.004$, $d=1.6)$. There was no time and treatment interaction $\left(P=0.210, \eta^{2}=0.2\right)$ or main effect for treatment for $[B G]\left(P=0.629, \eta^{2}<0.1\right)$. However, there was a 
main effect for time $\left(P<0.001, \eta^{2}=0.9\right)$. At the end of exercise $(3.68 \pm 0.22$ mmol..$\left.^{-1}\right)$ and 5 min post-exercise $\left(3.54 \pm 0.26 \mathrm{mmol}^{-1} \mathrm{I}^{-1}\right)[B G]$ was lower than at rest $\left(4.64 \pm 0.40 \mathrm{mmol}^{-1}{ }^{-1}\right.$; both $\left.\mathrm{P} \leq 0.001 ; \mathrm{d}>3\right)$. There was no difference between treatments for HR although HR increased linearly over time. At the end of exercise HR for SHAM $\left(174 \pm 9 \mathrm{bpm}^{-1}\right)$ and PLA $\left(173 \pm 13 \mathrm{bpm}^{-1}\right)$ was greater than at rest $\left(77 \pm 9 \mathrm{bpm}^{-1}\right.$ and $79 \pm 7 \mathrm{bpm}^{-1}$, respectively, both $\mathrm{P}<0.001$, $d>9$ ). 
Discussion

This study evaluated the effect of providing pre-exercise information designed to increase expectancy associated with $\mathrm{NaHCO}_{3}$ ingestion on high intensity cycling capacity in recreationally active males. In support of our

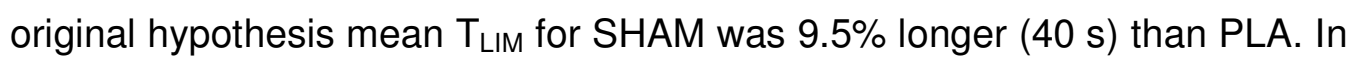
terms of contextualising the magnitude of this change, this is considered a moderate improvement. More simply the effect size of 0.3 sits between the 0.2 (small) and 0.5 (medium) benchmarks as originally suggested by Cohen (1988). Interestingly, this magnitude of improvement is in line with the range of effect sizes (mean: $0.69 ; 95 \% \mathrm{Cl}:-0.07-1.63$ ) for untrained individuals who have actually ingested $\mathrm{NaHCO}_{3}$ prior to exercise using a time to volitional fatigue (TLIM) protocol (Peart et al. 2012).

Beedie et al. (2007) suggest that placebo effects in sports performance are likely when individuals believe that they have ingested an ergogenic substance. Similar to McClung and Collins (2007), manipulative scripts were used in the present study to influence participant perception as to what they would be ingesting. Additionally, SHAM was mixed with carbonated water to try and replicate the reported side effects of $\mathrm{NaHCO}_{3}$ ingestion. Although symptoms were mild, in the present study gut fullness increased by $\sim 1$ unit for all trials post SHAM ingestion compared with PLA suggesting this strategy might have contributed to any increase in expectancy experienced by participants. It is important to highlight that we purposefully chose to not replicate the experimental design as adopted by 
McClung and Collins (2007). Due to the well acknowledged variety and sometimes severe Gl responses (Cameron et al. 2010; Carr et al. 2011) it was felt that actually administering $\mathrm{NaHCO}_{3}$ to participants could render the SHAM treatment ineffective in enhancing expectancy due to participants associating more severe $\mathrm{Gl}$ symptoms with 'genuine' $\mathrm{NaHCO}_{3}$ ingestion (Cameron et al. 2010). As such by comparing only PLA and SHAM, whose only differences were the information given pre-exercise and whether the water was tap or carbonated, we have been able to isolate the effects of preexercise information designed to increased expectancy of ergogenicity with $\mathrm{NaHCO}_{3}$ ingestion on exercise capacity. It appears that this strategy has contributed to the observed increase in exercise capacity, at least in some individuals.

Compared with PLA RPE $E_{0}$ was $1.4 \pm 1.3$ units lower for SHAM after 3 min $T_{\text {LIM }}$ with the associated effect size (0.6) equivalent to a medium to large magnitude of change (Cohen 1988). Moreover, both $R P E_{O}$ and $R P E_{L}$ were generally lower for SHAM versus PLA throughout $T_{\text {LIM. }}$. These results suggest that the information given to participants prior to exercise might have led to reduced perception of effort during exercise for SHAM which is likely to have contributed to the increase in $\mathrm{T}_{\text {LIM }}$. Our results are supported by McClung and Collins (2007) who reported that RPE (based on perceptions of lactate accumulation and therefore most similar to $\mathrm{RPE}_{\mathrm{L}}$ in the present study) was 1.5 units lower for the told $\mathrm{NaHCO}_{3} /$ given placebo trial compared to the told placebo/given placebo trial with the told $\mathrm{NaHCO}_{3} /$ given placebo trial performing the $1 \mathrm{~km}$ time trial $-2.8 \mathrm{~s} /-1.5 \%$ faster. Interestingly, both results 
are similar to Higgins et al. (2013) who reported RPE $E_{L}$ was 1.5 units lower after both 1 and 2 min of $\mathrm{T}_{\mathrm{LIM}}$ cycling at $100 \% \mathrm{~W}_{\text {PEAK }}$ after $\mathrm{NaHCO}_{3}$ ingestion. Moreover, the authors reported significant correlations between $\mathrm{T}_{\text {LIM }}$ and $\mathrm{RPE}_{\mathrm{L}}$ at the same time points. Therefore, it is plausible that reductions in RPE and any impact on exercise performance are not solely due to $\mathrm{NaHCO}_{3}$ induced mediation of acid-base balance (i.e. the pharmacological effects). These results are in accordance with the psychobiological model which suggests that reduced perception of effort can increase exercise performance (Ross et al. 2015; Smirmaul, et al. 2013) with perception of effort postulated as a key determinant of exercise tolerance (Marcora and Staiano 2010). The reduction in perception of effort might be linked to the mediation of endogenous opioids via expectancy orientated placebo effects (Benedetti et al. 2005).

In the present study the mean $9.5 \%(+40 \mathrm{~s})$ increase in exercise capacity after SHAM is substantially larger than the $-1.5 \%\left(\begin{array}{lll}-2.8 \mathrm{~s}\end{array}\right)$ improvement for the placebo/told $\mathrm{NaHCO}_{3}$ trial compared with the given placebo/told placebo trial reported by McClung and Collins (2007). This is likely due to a number of methodological differences between studies such as exercise protocol (capacity test vs. time trial), exercise modality (cycling ergometry vs. track running), participant training status (recreationally active vs. trained), placebo dosage ( 0.1 g.kg ${ }^{-1}$ vs. "pinch" of $\left.\mathrm{NaCl}\right)$, volume $(5 \mathrm{ml} . \mathrm{kg}$ ${ }^{1}$ vs. standardised $750 \mathrm{ml}$ ) and choice of solvent (carbonated water vs. (presumably) tap water) used to mix experimental solutions. However, it seems clear from both studies that deceptive information given pre-exercise 
with regards to $\mathrm{NaHCO}_{3}$ ingestion has subsequently improved performance when compared with placebo.

Despite the expected increases in [BLa] and HR and decreases in [BG] from rest to post-exercise, respectively, in the present study there were no interactions or main effects for treatment for any of the cardiorespiratory or haematological data collected. The lack of difference between treatments

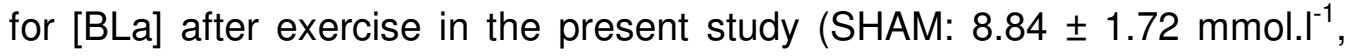
PLA: $8.99 \pm 1.79 \mathrm{mmol}^{-I^{-1}}$ ) is in accordance with McClung and Collins (2007). Taken together, these data suggest that these physiological variables did not contribute to the observed differences in $T_{\text {LIM. }}$.

To the best of our knowledge the daily variation for $\mathrm{T}_{\text {LIM }}$ at $100 \%$ $W_{P E A K}$ in recreationally active participants is currently unknown. Therefore, we have compared participant's individual data to the daily variation of $\sim$ $11 \% / 24 \mathrm{~s}$ reported for participants of a similar training status who engaged in two bouts of $T_{\text {LIM }}$ cycling at $110 \% W_{\text {PEAK (Higgins et al. 2014). In the }}$ present study five of the eight participants increased $\mathrm{T}_{\text {LIM }}$ for SHAM above daily variation (range: $12 \%$ to $32 \%$; 53 to 104 s) with two participants showing no difference (range: $-2 \%$ to $3 \%$; -10 to 14 s) with only one recording lower TLIM for SHAM (-9\%; -39s). It is unclear why expectancy appears to have influenced some but not all individuals but it is plausible that individuals might respond differently to expectancy at different exercise intensities. 
It is important to acknowledge that there are some limitations to our study. Firstly, the present study examined the effects of expectancy related to an oral bolus solution ingested $60 \mathrm{~min}$ prior to exercise. This method was chosen because $\mathrm{NaHCO}_{3}$ based solutions are widely used in contemporary research (Higgins et al. 2013; McClung and Collins 2007; Price and Simons 2010). In contrast $\mathrm{NaHCO}_{3}$ is also often administered via capsules and/or ingestion is staggered over multiple dosages (Carr et al. 2011; Krustrup et al. 2015). Further research is warranted to examine whether these ingestion methods report results similar to the present study. Secondly, although limited anecdotal evidence was recorded (e.g. participant one left the lab stating "that stuff definitely works!"; analysis after the study revealed this was for a SHAM trial which translated into a $65 \mathrm{~s} / 13 \%$ increase compared with PLA) we did not carry out post-hoc qualitative analysis which could have provided important information to verbally confirm the presence or not of expectancy at an individual level. Finally, as the placebo effect is a psychobiological phenomenon that is linked to different mechanisms including Pavlovian conditioning and expectation of improvement, it is important to acknowledge that there is no singular placebo effect. As such, different mechanisms might be responsible in different conditions (Benedetti et al. 2005). That said, according to McClung and Collins (2007) the vast majority of placebo effects are due to expectancy and in the context of our study design it seems likely expectancy has played at least some role in facilitating improvements in TLIM. 
From a practical application perspective, the results from this study suggest that athletes and coaches should consider psychobiological strategies which could positively impact physical training and/or performance to a similar extent as reported pharmacological agents. This might be especially important for individuals who have empirical evidence that $\mathrm{NaHCO}_{3}$ induced $\mathrm{Gl}$ distress negatively impacts their performance. Indeed, the development of psychological skills can be as important as physiological adaptations when looking to improve sport and exercise performance (Beedie and Foad 2009).

In conclusion, providing pre-exercise information designed to increase expectancy associated with $\mathrm{NaHCO}_{3}$ ingestion, administered via an oral bolus solution, improved mean cycling capacity at 100\% W PEAK $_{\text {by }} \sim 10 \%$. In conjunction with the psychobiological model of exercise performance the increase in exercise capacity was likely facilitated by reduced perception of overall effort, possibly due to mediation of endogenous opioids via expectancy orientated placebo effects. The observed ergogenicity with SHAM did not affect our measures of cardiorespiratory physiology or metabolic flux. 
Conflict of interest statement

The authors declare that there is no conflict of interest associated with this study. 


\section{References}

Aschenbach, W., Ocel, J., Craft, L., Ward, C., Spangenburg, E., \& Williams, J. 2000. Effect of oral sodium loading on high-intensity-arm ergometry in college wrestlers. Med. Sci. Sport. Exer. 32(3): 669-674.

Beedie, C.J., \& Foad, A, J. 2009. The placebo effect in sports performance: a brief review. Sports. Med. 39(4): 313-329.

Beedie, C.J., Coleman, D.A., \& Foad, A.J. 2007. Positive and Negative Placebo Effects Resulting From the Deceptive Administration of an Ergogenic Aid. Int. J. Sport. Nutr. Exerc. Metab. 17(3): 259-269.

Benedetti, F., Mayberg, H.S., Wager, T.D, Stohler, C.S., \& Zubieta, J-K. 2005. Neurobiological mechanism of the placebo effect. J. Neurosci. 24(45): 10390-10402.

Borg, G.A.V. 1982. Psychophysical bases of perceived exertion. Med. Sci. Sport. Exer. 14(5): 377-381.

Broatch, J.R., Petersen, A., \& Bishop, D.J. 2014. Post exercise Cold-Water Immersion Benefits Are Not Greater than the Placebo Effect. Med. Sci. Sport. Exer. 46(11): 2139-2147. 
Cameron, S.L., McLay-Cooke, R.T., Brown, R.C., Gray, A.R., \& Fairbairn, K. A. 2010. Increased blood pH but not performance with sodium bicarbonate supplementation in elite rugby union players. Int. J. Sport. Nutr. Exerc. Metab. 20(4): 307-321.

Carr, A.J., Slater, G.J., Gore, C.J., Dawson, B., \& Burke, L.M. 2011. Effect of Sodium Bicarbonate on $\left[\mathrm{HCO}_{3}^{-}\right], \mathrm{pH}$, and Gastrointestinal Symptoms. Int. J. Sport. Nutr. Exerc. Metab. 21: 189-194.

Clark, V.R., Hopkins, W.G., Hawley, J.A., \& Burke, L.M. 2000. Placebo effect of carbohydrate feeding during a 40-km cycling time trial. Med. Sci. Sport. Exer. 32(9): 1642-1647.

Cohen, J. 1988. Statistical Power Analysis for the Behavioural Sciences. Routledge Academic, New York.

Derave, W., Everaert, I., Beeckman, S., \& Baguet, A. 2010. Muscle carnosine metabolism and $\beta$-alanine supplementation in relation to exercise and training. Sports. Med. 40(3): 247-263.

Evans, D. 2003. Placebo: The Belief Effect. Harper Collins, London.

Fernandes, A.L., Lopes-Silva, J.P., Bertuzzi, R., Casarini, D.E., Arita, D.Y., Bishop, D.J., et al. 2014. Effect of Time of Day on Performance, Hormonal 
and Metabolic Response during a 1000-m Cycling Time Trial. PLOS ONE. 9(10): 1-9. doi: 10.1371/journal.pone.0109954

Greenhaff, P. L., Gleeson, M., \& Maughan, R. J. 1988. Diet-induced metabolic acidosis and the performance of high intensity exercise in man. Eur. J. Appl. Physiol. O. 57(5): 583-590.

Higgins, M. F., James, R.S., \& Price, M.J. 2014. Familiarisation to and reproducibility of cycling at $110 \%$ peak power output. J. Sport. Med. Phys. Fit. 54(2):139-46.

Higgins, M. F., James, R.S., \& Price, M.J. 2013. The effects of sodium bicarbonate $\left(\mathrm{NaHCO}_{3}\right)$ ingestion on high intensity cycling capacity. J. Sports. Sci. 31(9): 972-981.

Kirsch, I. 1985. Efficacy expectations or response predictions: The meaning of efficacy ratings as a function of task characteristics. J. Pers. Soc. Psychol. 42(1): 132-136. doi: 10.1037/0022-3514.42.1.132

Krustrup, P., Ermidis, G., \& Mohr, M. 2015. Sodium bicarbonate intake improves high-intensity intermittent exercise performance in trained young men. J. Int. Soc. Sports. Nutr. 4: 12-25. doi: 10.1186/s12970-015-0087-6

Lamberts, R.P., Lambert, M.M., Swart, J., \& Noakes, T.D. 2012. Allometric scaling of peak power output accurately predicts time trial performance and 
maximal oxygen consumption in trained cyclists. Brit. J. Sports. Med. 46(1): $36-41$.

Lavender, G., \& Bird, S.R. 1989. Effect of sodium bicarbonate ingestion upon repeated sprints. Brit. J. Sports. Med. 23(1): 41-45.

Maganaris, C.N., Collins, D., \& Sharp, M. 2000. Expectancy effects of strength training: Do steroids make a difference? Sport. Psychol. 14(3): 272278.

Marcora, S. M., \& Staiano, W. 2010. The limit to exercise tolerance in humans: mind over muscle? Eur. J. Appl. Physiol. 109(4): 763-70. doi: $10.1007 / \mathrm{s} 00421-010-1418-6$

McClung, M., \& Collins, D. 2007. "Because I know it will!" Placebo effects of an ergogenic aid on athletic performance. J. Sport. Exercise. Psy. 29(3): 382-394.

Nakagawa, S., \& Cuthill, I.C 2007. Effect size, confidence interval and statistical significance: a practical guide for biologists. Biol. Rev. 82(4), 591605.

Parkhouse, W. S., McKenzie, D. C., Hochachka, P. W., \& Ovalle, W. K. 1985. Buffering capacity of deproteinized human vastus lateralis muscle. J. Appl. Physiol. 58(1): 14-17. 
Parkhouse, W. S., \& McKenzie, D. C. 1984. Possible contribution of skeletal muscle buffers to enhanced anaerobic performance: a brief review. Med. Sci. Sport. Exer. 16(4): 328-338.

Peart, D.J., Siegler, J.C., and Vince, R.V. 2012. Practical recommendations for coaches and athletes: a meta-analysis of sodium bicarbonate use for athletic performance. J. Strength. Cond. Res. 26(7): 1975-1983.

Price, M.J., \& Simons, C. 2010. The Effect of Sodium Bicarbonate Ingestion on High-Intensity Intermittent Running and Subsequent Performance. J. Strength. Cond. Res. 24(7): 1834-1842.

doi:10.1519/JSC.0b013e3181e06e4a

Ross, R., Gray, C.M., \& Gill, J.M. 2015. The Effects of an Injected Placebo on Endurance Running Performance. Med. Sci. Sport. Exer. 47(8): 1672-81. doi: 10.1249/MSS.0000000000000584

Schmider, E., Ziegler, M., Danay, E., Beyer, L., \& Bühner, M. 2010. Is It Really Robust? Reinvestigating the robustness of ANOVA against violations of the normal distribution assumption. Meth. Eur. J. Res. Meth. Behav. Soc. Sci. 6(4): 147-151.

Smirmaul, B. P. C., Dantas, J.L., Nakamura, F.Y., \& Pereira, G. 2013. The psychobiological model: a new explanation to intensity regulation and 
(in)tolerance in endurance exercise. Rev. Bras. de. Educ. Física. Esporte.

27: 333-340.

Tan, F., Polglaze, T., Cox, G., Dawson, B., Mujika, I., \& Clark, S. 2010. Effects of Induced Alkalosis on Simulated Match Performance in Elite Female Water Polo Players. J. Strength. Cond. Res. 20(3): 198-205.

Watt, K. K.O., Hopkins, W. G., \& Snow, R. J. 2002. Reliability of performance in repeated sprint cycling tests. J. Sci. Med. Sport. 5(4): 351-361. doi:10.1016/S1440-2440(02)80024-X

Wittekind, A. L., Micklewright, D., \& Beneke, R. 2011. Teleoanticipation in allout short-duration cycling. Brit. J. Sports. Med. 45(2): 114-119. 
Figure Legends

Figure 1 - Individual time until volitional exhaustion ( $T_{\text {LIM }}$ s) at $100 \% \mathrm{~W}_{\text {PEAK }}$ for SHAM vs. PLA. SHAM > PLA $(P=0.048, d=0.3)$.

Figure 2 - Perceptual ratings of gut fullness (GF) over time for SHAM vs. PLA. Error bars represent $1 \mathrm{SD}\left({ }^{\mathrm{a}}=\mathrm{SHAM},{ }^{b}=\mathrm{PLA}\right) .{ }^{*}$ Post-ingestion $>$ Rest $(\mathrm{P}=0.002),{ }^{\#}$ Post-ingestion $>$ end of exercise $(\mathrm{P}=0.031),{ }^{\$}$ Post-ingestion $>5$ min post-exercise $(\mathrm{P}=0.012),{ }^{* *}$ Pre-exercise $>5 \mathrm{~min}$ post-exercise $(\mathrm{P}=0.025)$.

Figure 3 - Ratings of perceived exertion representative of cardiovascular strain (RPEO) over time for SHAM vs. PLA. Error bars represent 1 SD. * SHAM $<$ PLA $(P=0.020)$. 


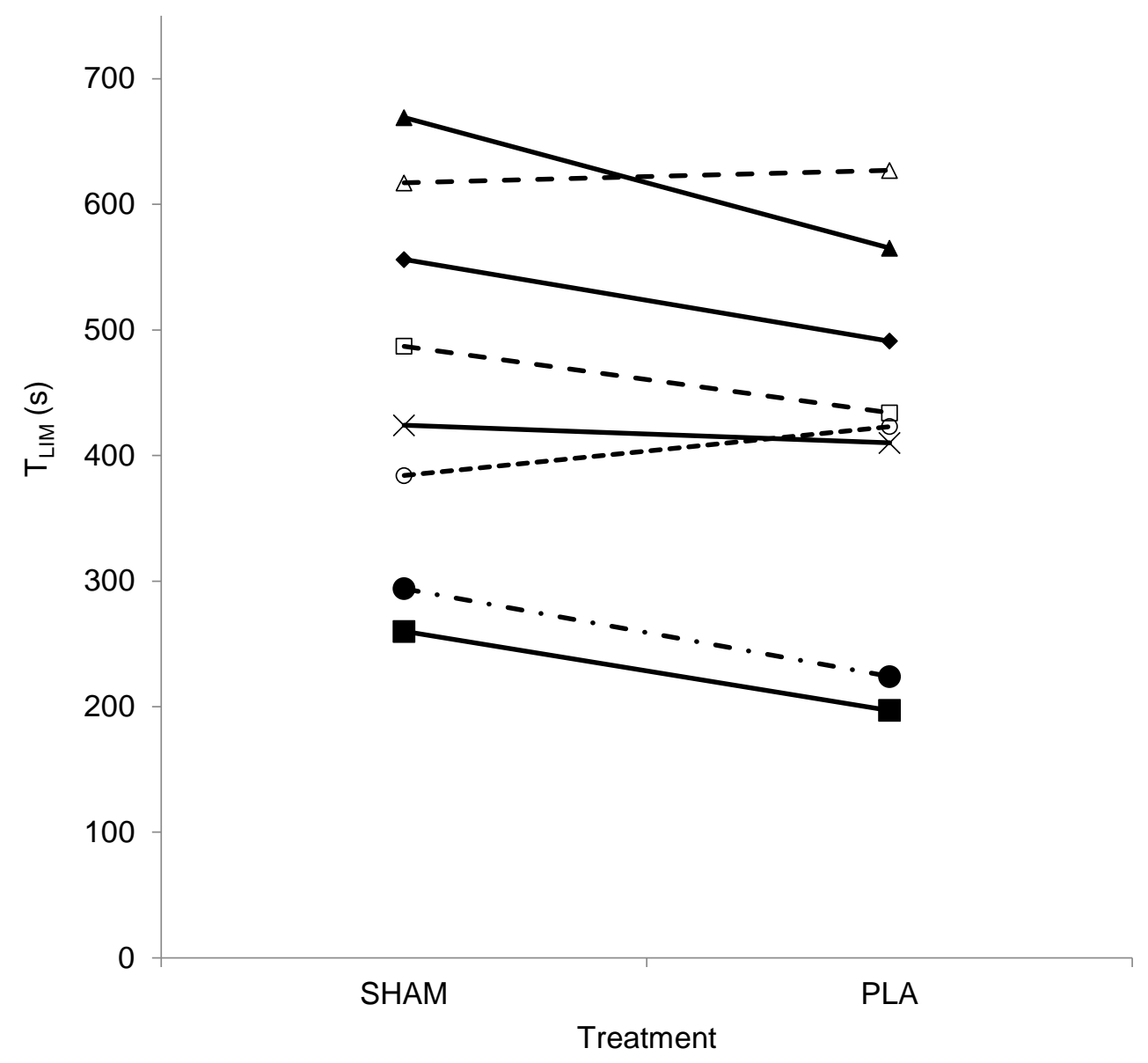




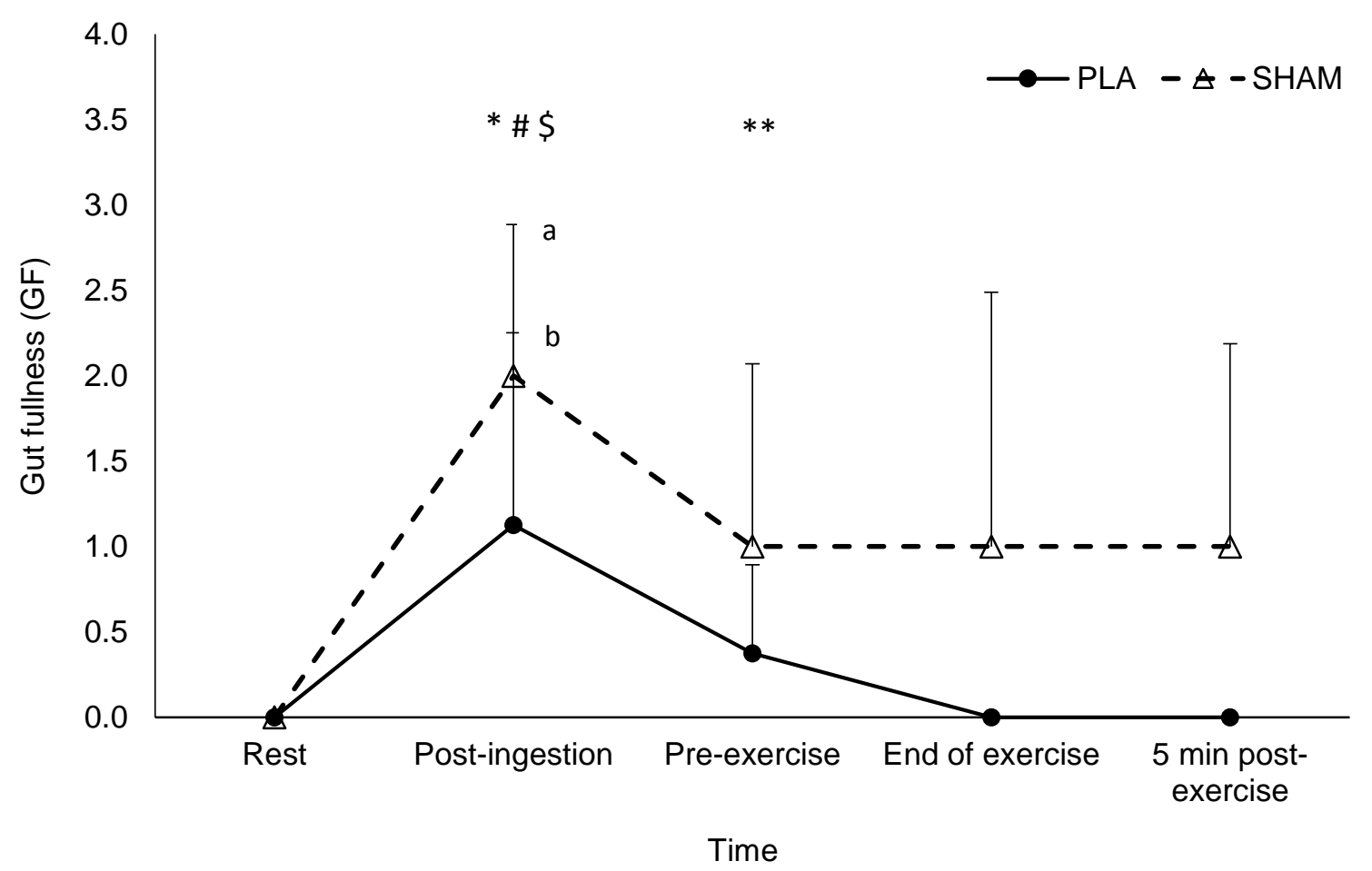




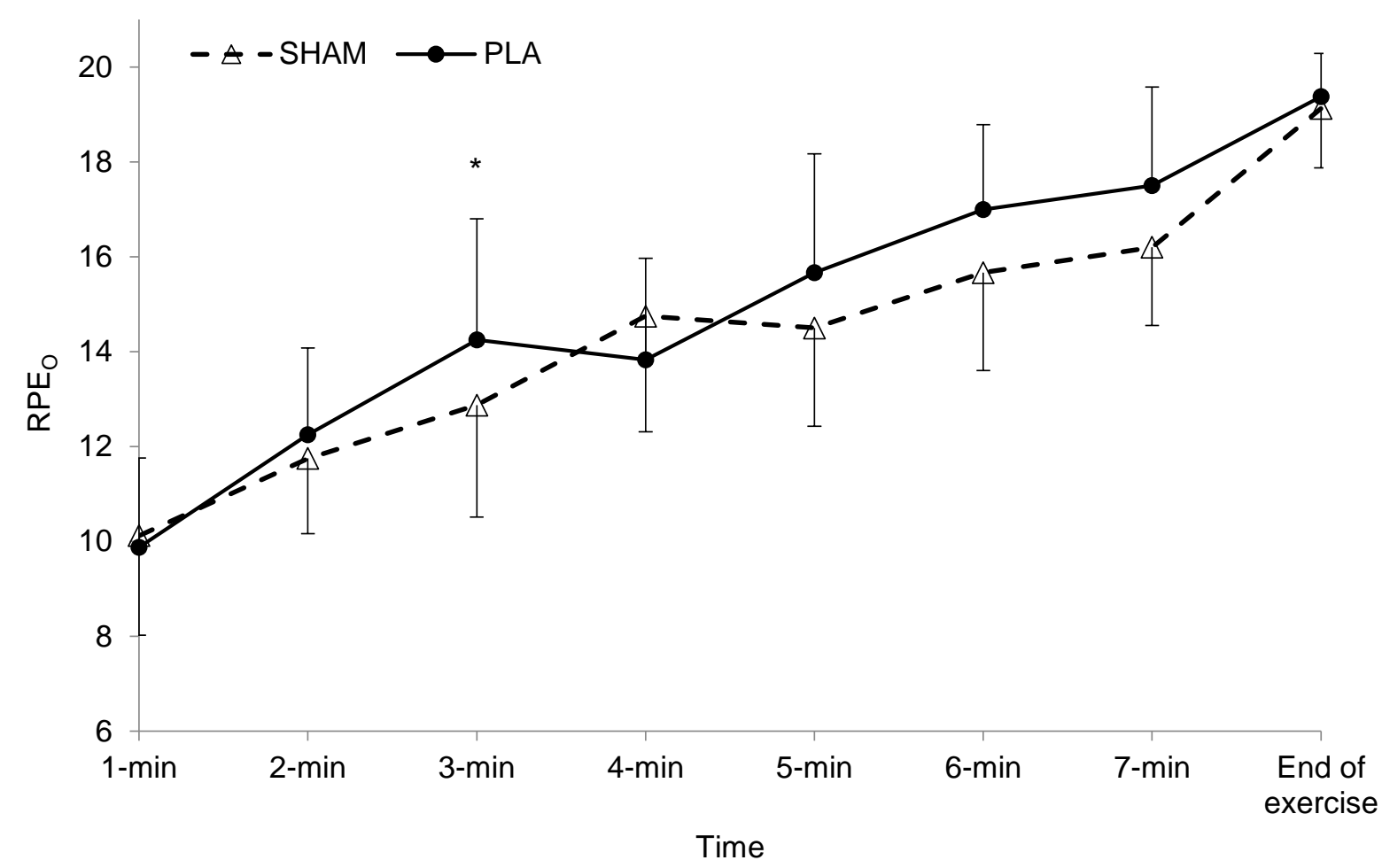

